\title{
Attentional capture triggers an attentional blink
}

\author{
WILLIAM S. MAKI and MICHAEL W. MEBANE \\ Texas Tech University, Lubbock, Texas
}

\begin{abstract}
Two experiments examined the possibility that attention directed to a distractor during rapid serial visual presentation (RSVP) can produce an attentional blink (AB). A to-be-ignored distractor (D1) preceded a target word (T2) by a variable lag in RSVP streams of black false-font distractors. D1 was highlighted by color and was a word, a string of consonants, a string of digits, or a string of false-font characters. Recall of T2 was significantly suppressed at short D1-T2 lags (the AB) but only when D1 contained letters; the $\mathrm{AB}$ was completely absent when $\mathrm{D} 1$ was composed of digits or false-font characters. Thus, the $\mathrm{AB}$ can be triggered by a highlighted distractor if the distractor shares features with a target.
\end{abstract}

The attentional blink $(\mathrm{AB})$ is a robust phenomenon observed in studies of visual attention that employ rapid serial visual presentation (RSVP). The AB is usually observed when two targets (T1 and T2) occur in close temporal proximity in the RSVP stream (see, e.g., Raymond, Shapiro, \& Arnell, 1992). Stimulus onset asynchronies (SOAs) are manipulated by varying the number of distractor items that intervene between the two targets. Identification of 2 is profoundly impaired, but the deficit is transient, with accuracy recovering gradually 200-600 msec after T1. Performance is usually perfect, or nearly so, in control trials in which observers are instructed to ignore $\mathrm{T} 1$ and report only $\mathrm{T} 2$, indicating that the $\mathrm{AB}$ does not arise merely from perceptual difficulties.

It is well known that the difficulty of spatial visual search depends on the similarity of targets and distractors (see, e.g., Duncan \& Humphreys, 1989). Studies of the AB using RSVP also indicate the importance of targetdistractor similarity for temporal visual search. Chun and Potter (1995), Maki and Padmanabhan (1994), and Maki, Couture, Frigen, and Lien (1997) have all shown that making targets distinct from the background stream of distractors attenuates the AB. Maki et al. (1997) presented two colored words as targets in streams of black words, strings of black consonants, or strings of black characters printed in a false font. An AB was found with word and consonant distractors but not with false-font distractors. The elimination of the $\mathrm{AB}$ with the false-font distractors was confirmed in a recent study by Maki, Bussard, Lopez, and Digby (2003); the AB was eliminated when two colored targets appeared in streams composed of false-font distractors. Maki et al. (2003) also showed a substantial

M.W.M is now at the Department of Psychology, Vanderbilt University. Experiments 1 and 2 were summarized at the meeting of the Psychonomic Society, Kansas City, MO, November 2002. We thank Jan Theeuwes and two anonymous reviewers for helpful comments on an earlier version of the manuscript. Correspondence concerning this article should be sent to W. S. Maki, Department of Psychology, Texas Tech University, Lubbock, TX 79409-2051 (e-mail: bill.maki@ttu.edu).
$\mathrm{AB}$ effect when the two target words were the same color (black) as the false-font distractors. To explain the difference between these results, Maki et al. (2003) argued that colored targets in the RSVP stream attracted attention more rapidly than did noncolored targets, thus allowing processing of the colored targets to commence sooner.

This argument, that colored targets attract attention, has an interesting implication. If color attracts attention in an RSVP stream (as it does in spatial cuing; Snowden, 2002), then colored distractors should attract attention. In studies of spatial attention, attention is often captured by salient distractors that may or may not be potential targets (see, e.g., Folk, Remington, \& Johnston, 1992; Theeuwes, Atchley, \& Kramer, 2000; Yantis, 2000). Thus, if the Maki et al. (2003) account of the effect of coloring targets is accurate, coloring a distractor in an RSVP stream should result in attention's being captured by the colored distractor. To the extent that the distractor possesses task-relevant features, disengagement of attention should be slowed (Theeuwes et al., 2000), resulting in an AB. The depth and duration of the resulting $\mathrm{AB}$ can be assessed by the accuracy of identifying a target that follows the colored distractor at varying SOAs.

The plausibility of triggering an AB by attentional capture is supported by a study by Folk, Leber, and Egeth (2002). Their observers viewed an RSVP stream of letters in which each letter was presented in one of five colors. The single target letter was printed in red. A critical distractor appeared prior to, concurrently with, or subsequently to the target. The critical distractor consisted of four eccentric symbols (\#s) arranged in a diamond-shaped array surrounding the central stream. Identification of the target was greatly reduced when the distractor preceded the target and one of the \#s was the same color as the target (red). This interference was interpreted as a spatial blink caused by contingent attentional capture (see Folk et al., 1992). Although this effect supports the general idea of a capture-induced $\mathrm{AB}$, it does not constitute a direct test of the prediction of an $\mathrm{AB}$ induced by a colored distractor within the RSVP stream. Folk et al. (2002) demonstrated a shift of attention away from the RSVP stream; the predic- 
tion considered here is that attention may be engaged by a distractor within the stream.

\section{EXPERIMENT 1}

The aim of the first experiment was to demonstrate an $\mathrm{AB}$ induced by a salient distractor that was part of the central, attended RSVP stream. Maki et al. (2003, Experiment 2) obtained a substantial AB effect when a target word was presented in the same color as the stream of false-font distractors. We presented those kinds of RSVP streams in this experiment with the addition of a critical distractor item that preceded the target. The lags (SOAs) between the distractor and the target covered the temporal range of the typical AB effect - approximately 200$600 \mathrm{msec}$. One type of critical distractor was a red word. A second type of critical distractor was a string of consonants, also printed in red. A third type of critical distractor was a false-font string presented in red. The fourth condition in the experiment was the control condition; the critical distractor was just another false-font string printed in black. These four conditions are illustrated in the leftmost four columns of Figure 1.

If attention were drawn to any colored event, then all three of the red distractor types should cause interference with reporting of the target. But if attention could be rapidly disengaged from distractors that did not resemble targets (Theeuwes et al., 2000), then only the red word and the red consonant string conditions should produce an AB.

\section{Method}

Participants and Instructions. College students enrolled in General Psychology (23 females and 4 males) were recruited and compensated with course credit. All of the participants reported (corrected) normal vision with no color defects. After we obtained informed consent, we described the RSVP task to the participants. They were told to ignore the distractors. The instructions were emphatic in describing the task as attending to and identifying the one black word and ignoring everything else.

Apparatus and Materials. The participants were tested individually using personal computers equipped with 15 -in. color monitors. Displays were synchronized with the refresh rate of the monitors $(75 \mathrm{~Hz})$. Stimuli were uppercase letters printed in 22-point Tahoma font and other characters printed in a false font (Maki et al., 2003;

\begin{tabular}{|c|c|c|c|c|}
\hline $\begin{array}{c}\text { Red-W } \\
\text { Lag } 2\end{array}$ & $\begin{array}{c}\text { Red-C } \\
\text { Lag } 3\end{array}$ & $\begin{array}{l}\text { Red-F } \\
\text { Lag } 4\end{array}$ & $\begin{array}{c}\text { Black-F } \\
\text { Lag } N\end{array}$ & $\begin{array}{c}\text { Red-D } \\
\text { Lag } 6\end{array}$ \\
\hline$\eta \mathbb{D} \pi \mathrm{Y}$ & $\eta \cap Y \Psi$ & $1 \# レ れ$ & HワVE & $\alpha \oslash \mathbb{D}$ \\
\hline 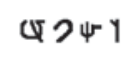 & ๑つரレ & $\Leftrightarrow 2 \|_{1} Y$ & $\mathbb{D} \cap \boldsymbol{X}$ & EYO \\
\hline ルNレO & IレルJ & $Y 517$ & Т๑ว & 5973 \\
\hline $20 \propto \#$ & CMMLB & ד ৩ & 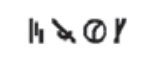 & $\pi r \supset \gamma$ \\
\hline$v \gamma \pi \pi$ & 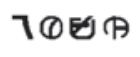 & 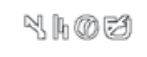 & $Q / \lambda \alpha$ & $y \oslash x \in$ \\
\hline PANTN & $Q J A Y$ & $\ln / Y J$ & JOIN & $5 \pi \mathbb{N}$ \\
\hline$\gamma \oslash 2 \mathbb{0}$ & NEWS & ? & YAOX & $O r / 7$ \\
\hline FEUD & ร2/1 & 1つ\#レ & フNA\# & $\mathbb{D} 1 \oplus \pi$ \\
\hline ANQT & $2 \sqrt{\mathbb{U}} \Psi$ & BEER & YつגE & MYTH \\
\hline$A X Q /$ & レフปヒ & $2 \Psi Y_{1}$ & $Q \cap E Y$ & $\alpha \times y$ \\
\hline $100 Y$ & ห 215 & $\mathbb{D}$ (AY 5 & & $E A Y \theta$ \\
\hline \multirow[t]{2}{*}{$\alpha \mathbb{Q} \in O$} & & $E Q Y J$ & & YYつA \\
\hline & & คつ $\Psi Q$ & & $2 \lambda Y A$ \\
\hline
\end{tabular}

Figure 1. Fragments of RSVP streams illustrating targets and distractors presented in Experiments 1 and 2. The target was a four-letter word printed in black. All other strings were to-be-ignored distractors, usually printed in black; when colored, the critical distractor was printed in red. Critical distractors were a red word $(\operatorname{Red}-W)$, a red consonant string (Red-C), a red false-font string (Red-F), a black false-font string (Black-F), or a red string of digits (Red-D). Each trial began with a variable number of false-font distractors, but every trial ended with four distractors following the target. Lag $N$ : Lag varies in that condition. 
Maki et al., 1997). The average letter and character subtended a visual arc of approximately $0.6^{\circ}$ (meaning that the typical four-letter word subtended an arc of about $2.4^{\circ}$ ) when viewed at $52 \mathrm{~cm}$, the distance between the participant and the monitor. Characters were printed in black or red on a gray background. ${ }^{1}$

For each participant, items for the RSVP lists were drawn at random and without replacement from three sets of stimuli: four-letter nouns and verbs $(N=524)$, strings of four randomly selected consonants $(N=994)$, and strings of four randomly selected vowels and consonants that were converted to false-font characters $(N=300)$. Characters were not repeated within consonant and false-font strings. Target words were assigned randomly to each distractor condition.

Procedure. Each trial consisted of a variable number of strings presented at the rate of $9.375 / \mathrm{sec}$. Most strings were distractors consisting of false-font characters printed in black. Two strings within each stream were critical events, a critical distractor and the target. The target was always a word presented in black. The critical distractor was either another black false-font string (Black-F), a red falsefont string $(\operatorname{Red}-F)$, a red consonant string $(\operatorname{Red}-C)$, or a red word $($ Red-W). Examples of these trials are shown in Figure 1.

Each session contained 208 trials. Each trial began with a fixation string (four "+" signs) presented for $506.7 \mathrm{msec}$. The fixation display was erased for $13.3 \mathrm{msec}$ prior to the presentation of the RSVP stream, which contained 9-16 strings of four characters. Each string was presented for $93.3 \mathrm{msec}$, followed by a $13.3-\mathrm{msec}$ blank frame. Trials differed with respect to distractor type (Black-F, Red-F, Red-C, or Red-W), distractor-target lag (D-T; 2, 3, 4, or $6)$, and distractor position $(3,4,5$, or 6$)$. At the end of each trial, a prompt to recall the target word was presented and the participant typed in a response.

Sessions were subdivided into one practice block containing 16 trials and three test blocks containing 64 trials each. Within each test block, each trial was uniquely defined by a combination of distractor type, D-T lag, and distractor position. The order of presentation was randomized within each block. Prior to beginning the experiment, the participants were reminded to watch for the one black word in each RSVP list and to ignore all other stimuli, regardless of color or form.

Data analysis. Data from the practice blocks were ignored. Data from the test blocks were scored with the aid of a computer program that matched as many response words as possible to their corresponding targets. Responses that failed to match were scored manually. Mismatches were judged to be typographical errors if the response was not a word and contained erroneous letters that were contiguous to correct letters on the keyboard (e.g., LONK vs. LINK, or LIM[ Vs. LIMP). Mismatches were judged to be spelling errors if they were nonwords that were phonologically correct (e.g., FUED vs. FEUD). Typographical and spelling errors were counted as correct responses; all other mismatches were counted as errors. Frequencies of correct responses were summed over the four distractor positions and the three test blocks, resulting in a maximum score of 12 correct for each combination of distractor condition and lag. The percentages of words correctly recalled were entered into the ANOVAs described below, and additional analytical tests were conducted as appropriate. The level of significance was set at .05 throughout.

\section{Results}

The essential results are shown in Figure 2. Recall of the target word was uniformly accurate when there was no preceding distractor (the Black-F condition). Recall was equally accurate when the preceding distractor was a highlighted false-font string (Red-F). However, when the distractor was a highlighted consonant string (Red-C), recall was diminished substantially at Lag 2 and recovered monotonically with increasing lag between the distractor and the target. This $\mathrm{AB}$ effect was even more pronounced when the distractor was a highlighted word (Red-W).

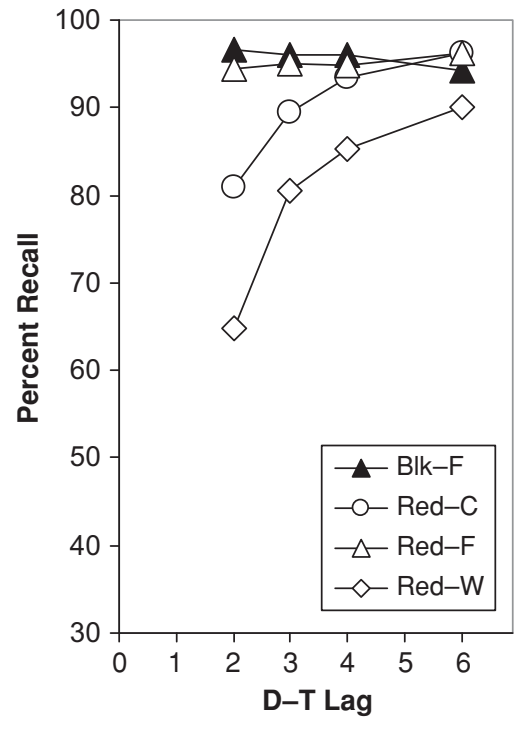

Figure 2. Recall accuracy in Experiment 1. The four curves in each panel correspond to the four conditions illustrated in the four leftmost columns of Figure 1. The critical distractor was a red word $(\operatorname{Red}-W)$, red consonant string $(\operatorname{Red}-\mathrm{C})$, red false-font string (Red-F), or just another black false-font string (Black-F).

The main effects of distractor type and D-T lag were reliable $\left[F(3,78)=15.71, M S_{\mathrm{e}}=5.12\right.$, and $F(3,78)=$ $23.14, M S_{\mathrm{e}}=1.28$, respectively]. On average, the Black$\mathrm{F}$ and Red-F distractor conditions did not differ $(F<1)$, but the recall in the Red-F condition exceeded that in the Red $-\mathrm{C}$ condition $\left[F(1,26)=13.59, M S_{\mathrm{e}}=0.74\right]$, and recall in the Red-C condition exceeded that in the Red-W condition $\left[F(1,26)=10.02, M S_{\mathrm{e}}=3.79\right]$.

The distractor type $\times \mathrm{D}-\mathrm{T}$ lag interaction was reliable $\left[F(9,234)=10.20, M S_{\mathrm{e}}=1.16\right]$. The increases in performance with increasing D-T lag were significant for both the Red-C $\left[F(3,78)=11.83, M S_{\mathrm{e}}=1.49\right]$ and the Red-W $\left[F(3,78)=23.08, M S_{\mathrm{e}}=2.02\right]$ conditions, but not for either of the other distractor types (both $F_{\mathrm{S}}<1$ ). The differences between the lag effects for the Red-C and Red-W conditions were reliable at each lag [smallest $t(26)=2.102]$, but the differences at shorter lags tended to be larger, as indicated by a distractor type $\times \mathrm{D}-\mathrm{T}$ lag interaction for these two distractor types $[F(3,78)=2.69$, $\left.M S_{\mathrm{e}}=1.33\right]$.

The colored word in the Red-W condition often intruded in recall. The average percentage of all errors resulting from these intrusions was $40.1 \%$. Numerous intrusions from distractors were also observed in the Red-C condition. Analysis of these intrusions is complicated by the qualitative variation in the observed transformations of consonant strings into words. Analysis of these kinds of errors was aggregated across experiments and is reported in Appendix A.

\section{Discussion}

Following Theeuwes et al. (2000), an explanation of the effects of the various distractors on target identification 
in terms of attentional capture begins with the claim that attention is drawn involuntarily to a colored item in the RSVP stream. But the duration of the attentional dwell time is determined by the extent to which the capturing item shares features with the target. Thus, attention drawn to the red word distractor resulted in a sizable $A B$ effect accompanied by a high incidence of red word intrusions in recall (in spite of the instructions to ignore all stimuli other than the one black word in each list). The same account apparently applies to processing of any wordlike distractor; although smaller in magnitude, the $\mathrm{AB}$ effect observed with consonant string distractors in the Red-C condition was significant. However, no AB effect at all was observed in the Red-F condition when one of the false-font distractors was highlighted. The absence of an AB effect in the Red-F condition suggests that color alone is not sufficient to produce a capture-induced $A B$, perhaps because the false-font string was so different from any target.

But the absence of an $\mathrm{AB}$ in the Red-F condition is not conclusive with respect to the importance of resemblance of distractor and target features for the capture-induced blink. The false-font characters are very different from letters in terms of rated familiarity and meaningfulness (Maki et al., 2003, Experiment 3). Thus, the failure to observe a capture-induced blink effect with the Red-F distractors in this experiment may have been due to the differences in familiarity and/or meaningfulness of the false-font characters. A stronger case could be made for a capture-induced blink if the contribution of these variables could be ruled out.

\section{EXPERIMENT 2}

This experiment pitted two hypotheses against one another. As noted above, a meaningfulness-familiarity hypothesis explains the lack of an AB effect in the Red-F condition of Experiment 1 by appealing to the conceptual differences between false-font characters and letters. The capture hypothesis attributes the lack of an $\mathrm{AB}$ effect to the differences between the false-font string and the specifications contained in the task set (searching for words that are composed of letters). To distinguish between the two hypotheses, we included a distractor that was highly familiar and meaningful and yet shared no specifications with the task set - a highlighted string of digits. Maki et al. (2003) showed that college students rated digits and letters as equally meaningful and equally familiar (in contrast to false-font characters, which were rated almost completely unmeaningful and unfamiliar). The meaningfulnessfamiliarity hypothesis predicts that distractors composed of red digits should show an $\mathrm{AB}$ effect equivalent to that shown by consonant strings, because digits are just as meaningful and familiar as letters. The capture hypothesis predicts that the red digit distractors should show no $\mathrm{AB}$ effect, because digits are not relevant to the task of identifying word targets.

\section{Method}

Experiment 2 was conducted like Experiment 1, with two exceptions. First, the red distractor words were replaced by strings of four randomly selected digits (Red-D); stimuli from an example trial are shown in the rightmost column of Figure 1. Second, the duration of each item in the RSVP stream was shortened by one refresh cycle $(13.3 \mathrm{msec})$, resulting in an RSVP rate of $10.7 / \mathrm{sec}$. College students were recruited from the same pool as in the preceding experiment (21 females and 9 males). Again, they were told to attend to only the one black word in each RSVP list.

The familiarity and meaningfulness of the different types of distractor strings were determined empirically by having college students rate words, consonant strings, digit strings, and distractors (see Appendix B for details).

\section{Results}

The results of this experiment are shown in Figure 3. As in Experiment 1, recall appeared uniformly accurate and varied little over lag in the Black-F and Red-F conditions. Also as in Experiment 1, a pronounced AB effect occurred for the Red-C distractors. Importantly for the attentional capture hypothesis, recall following Red-D distractors was virtually indistinguishable from the recall in the two false-font distractor conditions.

Both main effects of distractor type and D-T lag were significant $\left[F(3,87)=66.00, M S_{\mathrm{e}}=2.17\right.$, and $F(3,87)=$ $19.77, M S_{\mathrm{e}}=1.07$, respectively]. The two false-font distractor conditions (Black-F and Red-F) did not differ $(F<1)$. The Red-F also did not differ reliably from the Red-D condition $(F<1)$. But accuracy of recall in the

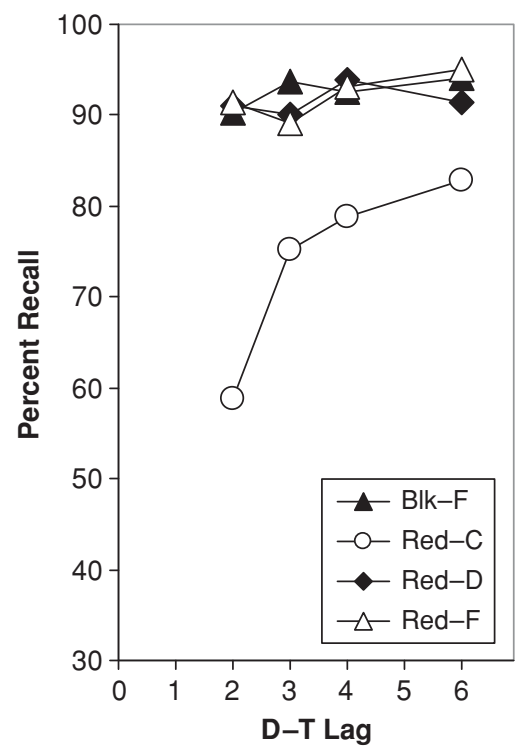

Figure 3. Recall accuracy in Experiment 2. The four curves correspond to the four conditions illustrated in the rightmost four columns of Figure 1. Three of the critical distractors were repeated from Experiment 1: the red consonant string ( $\operatorname{Red}-\mathrm{C})$, the red false-font string (Red-F), and the black false-font string (Black-F). The fourth critical distractor was a string of red digits (Red-D). 
Red-D condition exceeded that in the Red-C condition $\left[F(1,29)=67.24, M S_{\mathrm{e}}=14.96\right]$.

The effects of type of distractor varied significantly across lag $\left[F(9,261)=10.58, M S_{\mathrm{e}}=1.00\right]$. It appears from Figure 3 that most of the interaction was a result of the pronounced effect of lag for the Red-C distractor in comparison with the smaller and less consistent variations over lags in the other three distractor conditions. The Black-F, Red-F, and Red-D conditions were entered into a separate analysis, with distractor and lag as variables. There was no overall difference due to distractor condition $(F<1)$, but there was significant variation due to lag $\left[F(3,87)=3.46, M S_{\mathrm{e}}=0.73\right]$. Although the interaction $\left[F(6,174)=2.13, M S_{\mathrm{e}}=0.60\right]$ tended toward statistical significance, the variations appeared small and difficult to interpret. In contrast, the lag effect for the Red-C condition was much more pronounced and resembled the typical blink effect (as in the corresponding Red-C functions in Figure 2, for example) $\left[F(3,87)=22.44, M S_{\mathrm{e}}=2.12\right]$.

As in Experiment 1, we observed many intrusions from red consonant distractors. These intrusions appeared to result from attempts to process the consonant string as if it were a word. Appendix A contains the details of this analysis.

\section{Discussion}

The outcome of this experiment was quite clear. Red consonant strings produced a capture-induced blink, but the red digit strings did not. The meaningfulnessfamiliarity hypothesis would have to explain this result by asserting greater meaningfulness and/or familiarity of consonant strings. However, ratings of familiarity and meaningfulness (Appendix B) show that consonant strings are actually less familiar and less meaningful than are digit strings. Thus, we conclude that the pattern of results was not a result of variations in meaningfulness and familiarity. Rather, it appears that distractors that do not share properties with the target (in this case, letters) do not produce a capture-induced $\mathrm{AB}$ even though those distractors may be made salient by color highlighting. One explanation for the absence of an $\mathrm{AB}$ in these circumstances is that attention is drawn to such distractors automatically (by the color; Snowden, 2002) but then is quickly disengaged and available for deployment to a subsequent target (Theeuwes et al., 2000). In contrast, attention drawn to a salient distractor that shares features with a target is disengaged more slowly, resulting in an $\mathrm{AB}$.

\section{GENERAL DISCUSSION}

The present set of experiments was performed with the aim of evaluating some conjectures about the existence and effects of attentional capture during RSVP. In explaining effects of target-distractor similarity on the AB, Maki et al. (2003) proposed that highlighting a target with color attracts attention to that target and allows its processing to commence sooner than for nonhighlighted targets. Maki et al. (2003) then offered the "possibility that [the AB] can be triggered by attentional capture" (p. 200).

Our results extend those of Folk et al. (2002); they showed that capture of attention by a distractor in a spatial location different from that of the target produced an $\mathrm{AB}$. Here, we showed that attentional capture by a distractor produces an $\mathrm{AB}$ even when the distractor and target appear in a common attentional location.

In this article, we reasoned that the degree of interference from a capturing distractor would depend on its sharing of features with a target (Theeuwes et al., 2000); it would be more difficult, we argued, to disengage attention from a distractor that resembled a target, thus prolonging an (inappropriate) attentional episode and causing an $\mathrm{AB}$. We found two signs of engagement of attention by a colored distractor. Some errors appeared to arise from processing the red consonant strings as if they were words (Appendix A); other errors arose from intrusions when the critical distractor was a colored word (Experiment 1). However, there was no sign that color alone produced such interference; a distractor composed of colored digits, for example, had no effect on performance. Thus, a colored singleton distractor in an RSVP stream appears to have attracted attention, but an $\mathrm{AB}$ was produced only to the extent that the distractor shared task-relevant features with the target.

Although the present results are consistent with the preceding account of attentional capture during RSVP, a different explanation is possible, based on previous studies of target-distractor similarity. For example, Ghorashi, Zuvic, Visser, and Di Lollo (2003, Experiment 7) presented RSVP streams of distractors. Each stream consisted of all single letters or all tilted lines. Each stream ended with a letter target $\left(C\right.$ or $G$ ) or a tilted line target $\left(45^{\circ}\right.$ or $135^{\circ}$ ). Reaction times were shorter in the distractor-target dissimilar conditions (line-letter or letter-line) than in the distractor-target similar conditions (line-line or letterletter). The interpretation of these results that Ghorashi et al. favored was cast in terms of capture of attention by distractors that shared features with the target. When, for example, the target was defined by its being a letter, the preceding letter distractors also commanded attention and thus produced an $\mathrm{AB}$ that slowed responses to the target. Applied to the present experiments, this account has attention being attracted by red word and red consonant distractors, not because they were highlighted by color, but only because they contained letters. The extent to which color highlighting in the present experiments contributed to the control of attention is a question that needs additional experimental work.

The present experiments answer another question posed by Ghorashi et al. (2003, p. 89): "given that targets are letters, it could be asked to what extent contingent capture would occur if the distractors were false fonts, or digits, or other stimuli that shared features with letters." In both of our experiments, only the critical (colored) distractors that contained letters produced an $\mathrm{AB}$; strings of false fonts 
and digits had no effect at all. Thus, although some of the details remain to be defined, the available evidence suggests that when distractors share features with targets, attentional capture by distractors does trigger an AB.

\section{REFERENCES}

Chun, M. M., \& Potter, M. C. (1995). A two-stage model for multiple target detection in rapid serial visual presentation. Journal of Experimental Psychology: Human Perception \& Performance, 21, 109-127.

Duncan, J., \& HumphreYs, G. W. (1989). Visual search and stimulus similarity. Psychological Review, 96, 433-458.

Folk, C. L., Leber, A. B., \& Egeth, H. E. (2002). Made you blink! Contingent attentional capture produces a spatial blink. Perception \& Psychophysics, 64, 741-753.

Folk, C. L., Remington, R. W., \& Johnston, J. C. (1992). Involuntary covert orienting is contingent on attentional control settings. Journal of Experimental Psychology: Human Perception \& Performance, 18, 1030-1044.

Ghorashi, S. M. S., Zuvic, S. M., Visser, T. A. W., \& Di Lollo, V. (2003). Focal distraction: Spatial shifts of attentional focus are not required for contingent capture. Journal of Experimental Psychology: Human Perception \& Performance, 29, 78-91.

Maki, W. S., Bussard, G., Lopez, K., \& Digby, B. (2003). Sources of interference in the attentional blink: Target-distractor similarity revisited. Perception \& Psychophysics, 65, 188-201.

Maki, W. S., Couture, T., Frigen, K., \& Lien, D. (1997). Sources of the attentional blink during rapid serial visual presentation: Perceptual interference and retrieval competition. Journal of Experimental Psychology: Human Perception \& Performance, 23, 1393-1411.

Maki, W. S., \& Padmanabhan, G. (1994). Transient suppression of processing during rapid serial visual presentation: Acquired distinctiveness of probes modulates the attentional blink. Psychonomic Bulletin \& Review, 1, 499-504.

Raymond, J. E., Shapiro, K. L., \& Arnell, K. M. (1992). Temporary suppression of visual processing in an RSVP task: An attentional blink? Journal of Experimental Psychology: Human Perception \& Performance, 18, 849-860.

SNOWDEN, R. J. (2002). Visual attention to color: Parvocellular guidance of attentional resources? Psychological Science, 13, 180-184.

Theeuwes, J., Atchley, P., \& Kramer, A. F. (2000). On the time course of top-down and bottom-up control of visual attention. In S. Monsell \& J. Driver (Eds.), Control of cognitive processes: Attention and performance XVIII (pp. 105-124). Cambridge, MA: MIT Press.

YANTIS, S. (2000). Goal-directed and stimulus-driven determinants of attentional control. In S. Monsell \& J. Driver (Eds.), Control of cognitive processes: Attention and performance XVIII (pp. 73-103). Cambridge, MA: MIT Press.

\section{NOTE}

1. Stimulus displays, timing, and response recording were accomplished with an experiment-generation package, Inquisit (www.millisecond.com). Inquisit uses an $\mathrm{R}-\mathrm{G}-\mathrm{B}$ color model in which the intensity of each primary color (red, green, blue) ranges from 0 to 255 . Red was defined as 255-0-0, gray was 200-200-200, and black was 0-0-0.

\section{APPENDIXA \\ Analyses of Errors: Target Confusions and Distractor Intrusions}

We examined 504 errors from the red consonant distractor conditions in Experiments 1 and 2. Responses that contained at least three letters from either the distractor or the target accounted for $45.6 \%$ of those errors. Of those three- and four-letter matches, $27.4 \%$ involved partial matches between the distractor and the response ("distractor intrusions"); the remainder involved partial matches between the target and the response ("target confusions"). In discussing the qualitative differences between errors, we will use the format distractortarget-response to present examples.

Target confusions. Some target confusions involved the addition of a letter that was not part of either the distractor or the target: RBWD-MOTH-MONTH, TBFW-HERD-HEARD. Other target confusions occurred because of transposition errors: DFCV-LIAR-LAIR. Some target confusions appeared to result from perceptual errors: XGZHPEST-REST. Other confusions involved the blending of letters from both distractor and target: GXTR-FOAM-FORM. The remaining target confusions could not be otherwise classified: BNFL-MARE-MARK, CNRM-THAW-HAWK.

Distractor intrusions. The reordering of letters and the introduction of a vowel were responsible for some distractor intrusions: $\mathrm{CMLB}-\mathrm{STAB}-\mathrm{CLIMB}$. Other distractor intrusions reflected use of some information from the target: SHMP-CROW-SHRIMP. Many of the intrusions used three-letter sequences from the distractor supplemented by a vowel: XSHP-CONE-SHIP, TSFK-CLUE-TASK. Some such cases involved exchanging a consonant for a vowel: CMSH-FORK-CASH, SPMN-CALL-SPIN. Yet other intrusions were based on some combination of these procedures: RCLD-BARN-CARD, DKSR-PILE-DISK.

The different kinds of errors described above, target confusions and distractor intrusions, seem qualitatively distinct. Target confusions appear to result from faulty processing of the target. Distractor intrusions appear to result from attempts to process the consonant-string distractor as if it were a word. Whether target confusions and distractor intrusions arise online during processing of the RSVP stream or offline during the attempt to recall the target cannot be determined from the present data. 


\section{Ratings of Familiarity and Meaningfulness of Distractor Strings}

Maki et al. (2003, Experiment 3) determined the familiarity and meaningfulness of single characters (letters, digits, and false-font characters). However, the familiarity and meaningfulness of strings of these characters may not be the same as it is for the single characters.

Random samples of 15 each were drawn from the pools of words, consonant strings, digit strings, and falsefont distractor strings used in Experiments 1 and 2. The resulting 60 items were arranged in 15 randomized blocks of 4 items each; each block contained 1 of each kind of item. Two rating forms were constructed, one being the reverse of the other; the two forms were used equally often.

The participants were 12 volunteers ( 5 males and 7 females) recruited from psychology graduate students $(n=4)$ and undergraduates $(n=8)$ who were members of the Psychology human subjects pool at Texas Tech University. The participants were asked to rate each of the 60 items on familiarity and meaningfulness using the 4-point scales (0-3) used by Maki et al. (2003, Experiment 3).

There was generally good agreement among raters on both measures. Interrater reliabilities for familiarity averaged $r=.76$ (range: .55-.98); reliabilities for meaningfulness averaged $r=.78$ (range: .42-1.00).

Average ratings for each of the four kinds of items are listed in Table B1. Also listed are the $95 \%$ confidence limits. Not surprisingly, the words were rated very high with respect to both familiarity and meaningfulness; the false-font strings are rated as totally unfamiliar and unmeaningful. The ratings for the consonant strings and digit strings, however, are of interest. The familiarity-meaningfulness hypothesis developed in the text would be forced to predict that consonant strings should be rated higher than digit strings. However, as Table B1 shows, the opposite pattern was observed; digit strings were rated significantly higher than were consonant strings on both familiarity and meaningfulness.

Table B1

Means and Confidence Intervals for Ratings of Familiarity and Meaningfulness

\begin{tabular}{|c|c|c|c|c|}
\hline \multirow[b]{2}{*}{ String } & \multicolumn{2}{|c|}{ Familiarity } & \multicolumn{2}{|c|}{ Meaningfulness } \\
\hline & Mean & $\mathrm{CI}$ & Mean & $\mathrm{CI}$ \\
\hline Word & 2.61 & $2.49-2.72$ & 2.72 & $2.62-2.81$ \\
\hline Digits & 1.01 & $0.96-1.06$ & 0.76 & $0.72-0.79$ \\
\hline Consonants & 0.54 & $0.43-0.64$ & 0.41 & $0.30-0.51$ \\
\hline False font & 0.08 & $0.08-0.08$ & 0.00 & $0.00-0.00$ \\
\hline
\end{tabular}

Note_-Values are given for lower and upper 95\% confidence intervals (CIs). A Tukey test indicated that every pairwise comparison between string types is significant for both familiarity and meaningfulness measures. 